\title{
Disfunçóes Sexuais em Pacientes Após Acidente Vascular Cerebral
}

\author{
Sexual Dysfunction In Patients Post-Stroke
}

\section{Ebe dos Santos Monteiro ${ }^{1}$, Marina Oliveira Zirpoli , Igor de Queiroz Issa ${ }^{2}$, Priscila Nunes Moreira ${ }^{2}$}

\section{RESUMO}

Objetivo. Analisar por meio de uma revisão da literatura, a interferência do acidente vascular cerebral (AVC) na sexualidade, e nas principais disfunçôes sexuais após a lesão. Método. Foi realizada uma revisão bibliográfica baseada em artigos científicos de revisão, ensaios clínico randomizados controlados, editoriais, guias práticos, relatos de caso. Foram incluídos estudos publicados de janeiro de 1999 a março de 2010, em inglês, português e espanhol. Resultados. Foram encontrados 380 artigos dos quais 6 entraram nos critérios de inclusão. Em todos os 6 estudos foi encontrado piora da qualidade de vida, em 5 foi visto que os paciente masculinos sofrem de disfunçáo erétil, em 4 alteraçôes transtorno do desejo sexual, em 3 problemas na ejaculaçáo, em 2 foi citado à diminuição da frequência e transtornos no orgasmo, apenas em 1 dor e aversão sexual como consequências do AVC. Conclusáo. $\mathrm{O}$ estudo verificou que pacientes após o AVC apresentam como sequelas disfunçóes sexuais, diminuição da sexualidade e piora da qualidade de vida. Não se sabe ao certo correlacionar o tipo de disfunção com o local acometido, sendo necessário estudos direcionados.

Unitermos. AVC, Fisioterapia, Reabilitação, Sexualidade.

Citaçáo. Monteiro ES, Zirpoli MO, Issa IQ, Moreira PN. Disfunções Sexuais em Pacientes Após Acidente Vascular Cerebral.

\begin{abstract}
Objective. Analyze through a literature review the interference of cerebrovascular accident (Stroke) on sexuality and on main sexual dysfunctions after the injury. Method. A bibliographic review was made based on scientific articles of revision, random controlled clinical essays, editorials, practical guides, case reports. The studies included were published from January 1999 up to March 2010, in English, Portuguese and Spanish. Results. 380 articles were found, from which 6 were in the inclusion standards. In all of the studies selected was found the worsening of life quality, 5 of them reported that male patients suffer of erectile dysfunction, 4 of them state changes in sexual desire disorder, 3 of them report ejaculation problems, 2 of them mention decreasing frequency and disorder on orgasms, and only one of them reports pain and sexual aversion as Stroke consequences. Conclusion. This study verified that patients after the Stroke present sequels such as sexual dysfunctions, decreasing sexuality and worsening of life quality. It is not known for sure how to correlate the type of dysfunction to the local stricken, which requires further studies.
\end{abstract}

Keywords. Stroke, Physiotherapy, Rehabilitation, Sexuality.

Citation. Monteiro ES, Zirpoli MO, Issa IQ, Moreira PN. Sexual Dysfunction In Patients Post-Stroke.
Trabalho realizado no Centro Universitário Sáo Camilo - CUSC, São Paulo-SP, Brasil.

1.Fisioterapeuta, Especialista em uroginecologia, Hospital São Paulo, São Paulo-SP, Brasil.

2.Fisioterapeuta, Centro Universitário São Camilo, Sáo Paulo-SP, Brasil.
Endereço para correspondência: Ebe dos Santos Monteiro Rua: Casemiro de Abreu, 754 casa 7 CEP: 03013-001 São Paulo-SP, Brasil

E-mail: ebemonteiro@ibest.com.br 


\section{INTRODUÇÃO}

O Acidente Vascular Cerebral (AVC) é um déficit neurológico (transitório ou definitivo) em uma área cerebral secundário a uma lesão vascular, usualmente súbito do funcionamento cerebral. Existem dois tipos de AVC, o hemorrágico (AVCh) que compreende a hemorragia subaracnóide (HSA), em geral decorrente da ruptura de aneurismas saculares congênitos e a hemorragia intraparenquimatosa (HIP), onde há a degeneração hialina de artérias intraparenquimatosas cerebrais. O AVC isquêmico (AVCi) é resultante da insuficiência de suprimento sanguíneo cerebral, podendo ser temporário ou permanente. Os dois tipos de AVC estão geralmente associados a hipertensão arterial sistêmica (HAS), cardiopatias e diabetes mellitus (DM). Uma vez ocorrida à interrupção da circulação arterial, uma série de alterações funcionais e estruturais surgirá no território acometido, porém áreas vizinhas com perfusão parcial manterão um funcionamento mesmo que anormal, das quais será o principal alvo no tratamento ${ }^{1}$.

O AVC no Brasil tem representado uma importante causa de óbito, acometendo tanto homens como mulheres em diferentes faixas etárias, a permanência de sequelas incapacitantes, impondo aos pacientes limitaçóes motoras, sensitivas, sensoriais, de compreensão e expressão dos pensamentos pode alterar a dinâmica da vida dessas pessoas, não só pelas sequelas físicas que restringem as atividades da vida diária e tornam-nas, muitas vezes, dependentes de terceiros para movimentar-se e agir com maior ou menor independência, mas também por comprometerem suas possibilidades de administrar a vida pessoal e familiar. Assim, pode-se afirmar que o AVC é uma doença crônica que causa incapacidade, deficiências e desvantagens ${ }^{2,3}$.

As disfunçôes sexuais podem ser classificadas de formas diferentes; levando em consideração a cronologia, ou seja, as disfunçôes são primárias quando acontecem sempre durante a vida do indivíduo, já as secundárias se iniciam após um período de vida sexual saudável. Ou a fenomenologia, que seria a disfunção sexual geral ou global que ocorre em todas as circunstâncias da vida, e a disfunção situacional, que ocorrem apenas em algumas situações específicas ${ }^{4}$.

Essas disfunções afetam homens e mulheres, im- pedindo-os de ter um relacionamento sexual funcional e satisfatório, resultantes de fatores psicossociais, culturais e orgânicos 5 .

Doenças neurológicas frequentemente alteram as respostas sexuais e o processamento dos estímulos sexuais, como impedir a excitação, diminuir ou aumentar o desejo, reduzir o ingurgitamento genital, ereção, ejaculação e orgasmo. Porém, apesar da alta incidência pouco se sabe quais transtornos são gerados na vida sexual dos pacientes após lesão ${ }^{6,7}$.

Apesar desta alta incidência de casos, ainda temos poucas informaçôes sobre o funcionamento da vida sexual dos pacientes, são escassas as informaçóes da vida sexual do ser humano como um todo, e isso se evidencia ainda mais quando se delimita o tema da vida sexual em pacientes com disfunçóes cerebrais ${ }^{7}$. São encontrados normalmente em pacientes com AVC problemas como a diminuição do libido, a frequência com que realizam atividade sexual, diminuição da lubrificação vaginal, o orgasmo feminino, disfunção erétil e ejaculação precoce nos homens ${ }^{8}$.

A reabilitação da sexualidade após o AVC torna-se uma necessidade emergente, visto que atualmente a sexualidade é um dos pilares da qualidade de vida. A fisioterapia tem um vasto campo de atuação nas disfunções sexuais com evidência científica de reabilitação, com utilização de cinesioterapia, biofeedback, eletroterapia e orientaçóes sobre anatomia pélvica e as disfunçôes sexuais ${ }^{9}$.

O objetivo deste trabalho é analisar por meio de uma revisão narrativa da literatura, a interferência do acidente vascular cerebral na sexualidade, e nas principais disfunçôes sexuais após a lesão.

\section{MÉTODO}

Foi realizada uma revisão bibliográfica baseada em artigos científicos de revisão, ensaios clínicos randomizados controlados, editoriais, guias práticos, relatos de caso. Pesquisados nas bases de dados LILACS, BIREME, MEDLINE. Os descritores utilizados foram AVC, fisioterapia, reabilitação e sexualidade, nos idiomas português, inglês e espanhol.

Foram excluídos estudos publicados anteriormente a janeiro de 1999, e onde a sexualidade/disfunção sexu- 
al não estivesse relacionada diretamente ao AVC. Foram incluídos aqueles publicados de janeiro de 1999 a março de 2010, abrangendo a sexualidade e o AVC em humanos maiores de 18 anos de idade.

Dessa forma, os estudos encontrados foram analisados pelos três autores de maneira qualitativa, comparativa e independente. Os artigos selecionados foram encontrados na íntegra para nâo correr o risco de deixar estudos importantes fora da revisáo e foram considerados relevantes aqueles que abrangiam os objetivos do trabalho.

\section{RESULTADOS}

Inicialmente foram cruzadas as palavras sexualidade e AVC, encontrando-se 135 artigos. Destes, foram incluídos 3 no trabalho, por melhor condizerem com o tema.

Em um segundo momento, as palavras sexualidade e reabilitação, foram cruzadas encontrando-se 222 artigos dos quais 1 foi incluído, por apresentar mais detalhadamente os problemas da sexualidade e sua possível reabilitação. Finalmente, foram cruzadas as palavras sexualidade, AVC e reabilitação, onde foram encontrados 23 artigos, destes, apenas 2 foram selecionados devido à escassez literária do tema abordado.

Como resultado final, foram selecionados 6 artigos de acordo com os critérios de inclusão. Conforme Tabela 1 .

Em nossos achado pode-se verificar como principais alteraçôes sexuais, diminuição da frequência sexual, transtornos no orgasmo, presença de dor, problemas na ejaculação, disfunção erétil, aversão sexual, transtorno do desejo sexual, além da piora na qualidade de vida desses pacientes. Conforme Gráfico 1.

\section{DISCUSSÃO}

No que diz respeito à idade ${ }^{10}$, quanto maior a idade do paciente, menor é o desejo sexual, e as funções erétil e ejaculatória são prejudicadas, sendo a idade um fator importante que diferencia as disfunçóes sexuais comparando com o AVC. Em contrapartida, a idade não foi um fator importante para a diminuição da atividade sexual ${ }^{11}$.

O tratamento do AVC é mais complicado em pacientes com idades mais avançadas, a incidência de pacientes com AVC com idade inferior a 55 anos é de $10 \%$, enquanto que em paciente com idade inferior a 45 anos é de 3,9\%, além de que o impacto da idade influencia também na qualidade de vida ${ }^{13-15}$.

Um estudo realizado em $2010^{16}$, mostra a prevalência do AVC em homens acima de 60 anos e da raça negra. Em $2001^{15}$ a incidência do AVC era maior em mulheres na faixa dos 41 ao 45 anos, e em homens acima dos 45 anos.

Com relação às disfunções sexuais, 40 a $45 \%$ das mulheres e 20 a $30 \%$ dos homens referem alguma queixa de disfunção sexual, sendo as doenças cardiovasculares, as desordem psicológicas e psiquiátricas os principais fatores de risco associados. Em um estudo realizado com 74 pacientes com idade média de 81 anos mostra que 18\% das mulheres e $41 \%$ dos homens são sexualmente ativos ${ }^{17}$.

O sexo e a sexualidade, são parte fundamental da natureza humana e por isso a sua importância ultrapassa as necessidades reprodutivas ${ }^{4,18,19}$.

Com relação às alterações da sexualidade e por consequência as disfunçóes sexuais, diversos autores ${ }^{6-8,10-12,20}$ realizaram estudos investigando a correlação dos problemas sexuais nos pacientes após AVC e encontraram no geral que todos estes pacientes relatam uma diminuição na função sexual e muitas vezes por temerem ter uma vida sexual ativa pós AVC, por uma limitaçáo propriamente dita da função sexual, ou por problemas físicos e/ou emocionais.

Ainda referindo-se a esses estudos ${ }^{6-8}$, estes relataram como principal motivo da perda da função sexual após o AVC a impotência sexual. Complementando este assunto um destes estudos realizado em $1999^{7}$, direcionou ainda que essa perda da função sexual está relacionada à falta de conhecimento da sexualidade entre os pacientes e os seus conjugues. Porém, acredita-se que o posicionamento do corpo e os movimentos durante o sexo possam ser os maiores responsáveis pela diminuição ou perda da função sexual ${ }^{6}$.

Estudos com animais têm demonstrado um desencadeamento de ereçóes/hipersexualidade após estimulo direto do sistema Temporo-Límbico. O autor relata que lesóes bilaterais dos lobos temporais resultam no comportamento hiper $\neg$ sexual entre outros desequilíbrios do comportamento. Lesões específicas do Sistema Límbico, como lesóes da área septal, também têm demonstrado levar ao aumento do comportamento sexual. $\mathrm{O}$ autor rela- 


\begin{tabular}{|c|c|c|c|}
\hline Autor/Ano & Tipo de Estudo & $\mathbf{N}$ & Observaçóes \\
\hline Bia $2008^{8}$ & $\begin{array}{c}\text { Revisão bibliográfica } \\
\text { narrativa }\end{array}$ & - & $\begin{array}{l}\text { As alterações após AVC são de caráter físico e cog- } \\
\text { nitivo e podem resultar disfunçôes sexuais como: } \\
\text { desejo hipoativo; aversão sexual; impotência; eja- } \\
\text { culaçáo precoce; transtorno do orgasmo; dor e des- } \\
\text { conforto após ejaculação, dispareunia e vaginismo. }\end{array}$ \\
\hline Jung et al., $2008^{10}$ & Ensaio clínico & 109 & $\begin{array}{l}\text { A função erétil teve grande diminuição no grupo } \\
\text { pós AVC comparado com o grupo controle. Na } \\
\text { maioria dos pacientes do grupo AVC, a frequên- } \\
\text { cia de relaçóes sexuais e desejo diminuíram. } 59,4 \% \\
\text { sentem falta de desejo sexual. Quando as lesóes são } \\
\text { na parte direita do cerebelo tem uma desordem na } \\
\text { ejaculação e nos gânglios da base (esquerdo) tem } \\
\text { uma diminuiçáo no desejo sexual. Concluíram que } \\
\text { o desejo sexual, funçáo erétil, função ejaculatória } \\
\text { sáo comprometidos pós AVC. }\end{array}$ \\
\hline Korpelainen et al., $1999^{7}$ & Estudo transversal & $\begin{array}{l}192 \text { pacientes e } 94 \\
\text { cônjuges }\end{array}$ & $\begin{array}{l}\text { A disfunçáo sexual e a insatisfaçáo sexual são co- } \\
\text { muns tanto em pacientes homens quanto mulheres } \\
\text { e seus cônjuges. Os fatores sociais e psicológicos } \\
\text { têm um grande impacto na funçáo sexual e não } \\
\text { qualidade de vida sexual. }\end{array}$ \\
\hline Ress et al., $2007^{6}$ & $\begin{array}{c}\text { Segundo artigo de uma } \\
\text { série de três }\end{array}$ & - & $\begin{array}{l}\text { A disfunção sexual raramente é consequência ex- } \\
\text { clusiva do AVC, esta associada também às questōes } \\
\text { psicossociais e depressão. Disfunçôes: impotência } \\
\text { masculina, diminuição da lubrificação vaginal. }\end{array}$ \\
\hline Giaquinto et al., $2003^{11}$ & Estudo quantitativo & 62 & $\begin{array}{l}\text { Nos resultados } 50 \% \text { dos pacientes não fizeram } \\
\text { mais atividade sexual, após o AVC. O declínio se- } \\
\text { xual é ainda maior nas mulheres. Nos homens no } \\
\text { foi observado maior stress e tristeza com relação à } \\
\text { atividade sexual pós o AVC. }\end{array}$ \\
\hline Thompson et al., $2009^{12}$ & $\begin{array}{l}\text { Estudo qualitativo, } \\
\text { individual }\end{array}$ & 16 & $\begin{array}{l}\text { Nesse estudo as alteraçóes causadas pelo AVC fo- } \\
\text { ram descritas pelos próprios pacientes, que relatam } \\
\text { a diminuição no desejo sexual, a falta de vontade de } \\
\text { cuidar da própria aparência e o fato de não se sen- } \\
\text { tirem a vontade ao fazer novas amizades, além da } \\
\text { dependência. Porém alteraçóes físicas, psicológicas, } \\
\text { sociais e emocionais, além da aparência física, fadi- } \\
\text { ga e concentração são "problemas" relatados pelos } \\
\text { entrevistados. }\end{array}$ \\
\hline
\end{tabular}

ta ter encontrado hipersexualidade depois de tumores do lobo temporal, de lesão septal ou de AVC do lobo temporal. Concluindo entâo um aumento excessivo do desejo sexual em pacientes com disfunção frontal e temporal ${ }^{21}$. Em $1991^{22}$ verificou-se uma associação entre transtorno cerebral do Lobo Temporal e hipoatividade sexual, enquanto foi menos comumente relatada uma associação com hiperatividade sexual. Entretanto, a mesma pesquisa menciona autores em seu estudo que relatam hiperexcitação em pacientes com acometimento do Lobo Temporal. Mas apesar deste achado de outros autores, em seu estudo ficou concluído que após o comprometimento do Lobo Temporal, eram comuns a hipossexualidade e o exibicio- nismo. Portanto não existem estudos em humanos que comprovem a relação entre a área do cérebro afetada com o tipo de disfunção sexual ${ }^{7,11}$.

Embora exista certa dificuldade em associar transtorno cerebral e alteraçôes sexuais, o inverso é verdadeiro, ou seja, pacientes com alteraçóes da sexualidade podem apresentar anormalidades do lobo temporal ${ }^{23}$.

Após o AVC pode ocorrer comprometimento do desejo sexual, da funçáo erétil e ejaculatória, sendo que a diminuição do desejo sexual esta ligada em sua maioria às causas psicológicas e a disfunção erétil e o distúrbio da ejaculação associam-se a causas orgânicas, como lesôes na parte direita do cerebelo e nos gânglios da base (esquerdo). 


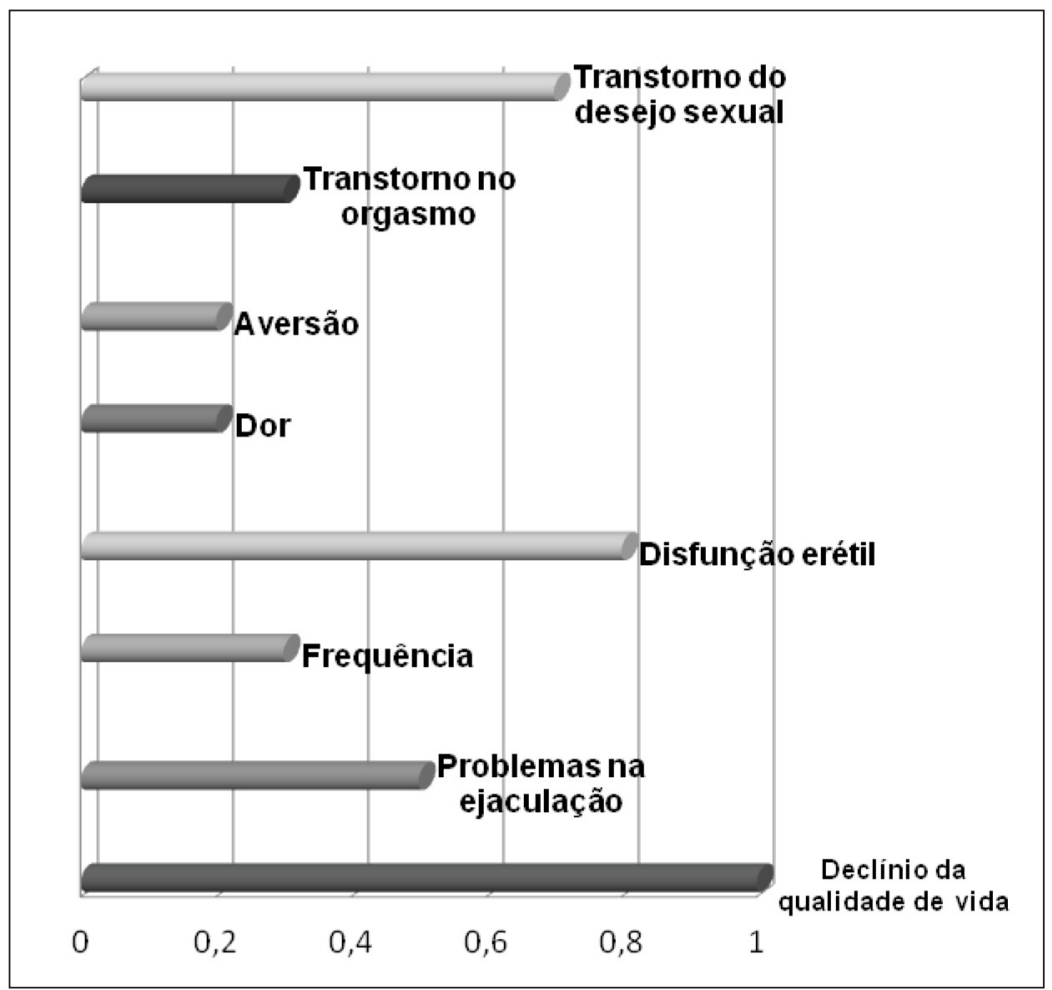

Gráfico 1. Alteraçôes sexuais após o AVC.

Em porcentagem do total dos artigos encontrados $(100 \%=1,00)$

Ainda neste estudo, concluíram que os pacientes que mostraram a lesão em muitas partes do cérebro tiveram uma importante diminuição da função erétil comparado com pacientes que mostram apenas lesões em uma área do cérebro ${ }^{10}$.

A avaliação da qualidade de vida (QV) deve ser realizada, levando em consideração decisóes de tratamento, políticas de pesquisa e fármacos ${ }^{7}$. A disfunção sexual diminui a qualidade de vida, bem como muda os hábitos pessoais dos indivíduos afetados ${ }^{10}$ e neste mesmo estudo aplicou o questionário SF-36 para avaliar a qualidade de vida em 96 pacientes, e mostrou que os scores dos mesmos demonstram diminuição da função, da satisfação e na qualidade de vida dos pacientes pós AVC.

Quanto maior o déficit da função física pior a qualidade de vida. A presença de depressão ou de seus sintomas também tem relação com a piora na QV. Artigos que abordaram idade e gênero mostram que o aumento da idade e do gênero feminino diminui ainda mais a $\mathrm{QV}^{14}$.

Em nossos achados nenhum autor cita o tratamento fisioterapêutico das disfunçóes sexuais nos pacientes pós AVC.
Porém, métodos como o uso de cones vaginais e contração voluntária visando à reeducação dos músculos do assoalho pélvico (MAP) e aumento do tônus, além de melhorar a conscientização da contração do assoalho pélvico. O biofeedback seria outra alternativa, pois tem efeito modulatório no Sistema Nervoso Central (SNC), onde ajuda a desenvolver maior percepção e controle voluntário dos MAP, sendo capaz de reestabilizar os circuitos neuronais e aperfeiçoar a função dos alvos periféricos. Essas técnicas tem mostrado resultados satisfatórios nas disfunçôes sexuais femininas (DSF), que podem advir de diversos fatores, porém os mais comuns são os psicológicos e o desconhecimento corporal, assim, as técnicas também parecem ser efetivas na conscientização perineal ${ }^{24}$.

\section{CONCLUSÃO}

Conclui-se que, a permanência de seqüelas após o AVC, impóe aos pacientes limitaçóes, podendo alterar a dinâmica de vida destes pacientes em diversas situaçóes, entre elas nas disfunçóes sexuais, na dependência de terceiros para mobilidade e atividades de vida diária, levando assim ao declíneo da qualidade de vida. 
Não se sabe ao certo correlacionar o tipo de disfunção sexual com o local da lesão cerebral, devido a poucos estudos nessa área. Sendo necessário o aprofundamento de pesquisas no que diz respeito a lesão cerebral e disfunção sexual, a fim de possibilitar a adequação do tratamento específico, visto que ainda é um campo pouco explorado.

\section{REFERÊNCIAS}

1.Radanovic M. Características do atendimento de pacientes com acidente vascular cerebral em hospital secundário. Arq Neuropsiquiatr 2000;58:99-106. http://dx.doi.org/10.1590/S0004-282X2000000100015

2.Lotufo PA, Bensor IM. Stroke mortality in São Paulo (1997-2003). A description using the tenth revision of the international classification of disease. Arq Neuropsiquiatr 2004;62:1008-11.

\section{http://dx.doi.org/10.1590/S0004-282X2004000600014}

3.Perlini NMOG, Faro ACME. Cuidar de pessoa incapacitada por acidente vascular cerebral no domicílio: o fazer do cuidador familiar. Rev esc enferm USP 2005;39:154-60.

http://dx.doi.org/10.1590/S0080-62342005000200005

4.Cabral R, Farta LCA. Sexualidade. In: Baracho E. Fisioterapia Aplicada à Obstetricia, Uroginecologia e Aspectos de Mastologia. Rio de Janeiro: Guanabara Koogan, 2007, p.270-9.

5.Martins PCR. Disfunçóes sexuais. Rev Ter Sex Clín Pesq Aspec psicossoc 2005;843-53.

6.Rees PM, Fowler CJ, Maas CP. Sexual function in men and woman with neurologic disorders. The Lancet 2007;369:512-25.

http://dx.doi.org/10.1016/S0140-6736(07)60238-4

7.Korpelainen JT, Nieminen P, Myllylã VV. Sexual function among stroke patient and their spouses. Stroke 1999;30:715-79.

http://dx.doi.org/10.1161/01.STR.30.4.715

8.Bia FMM. Reabilitação da sexualidade após Acidente Vascular Cerebral: esperança para o amor. Rev Nursing 2008;234:45-56.

9.Moreno AL. Fisioterapia em Uroginecologia. 2a . ed. São Paulo: Manole, 2009, 226p.

10.Jung JH, Kam SC, Choi SM, Jae SU, Lee SH, Hyun JS. Sexual dysfunction in male stroke patients: Correlation between brain lesions and sexual function. Urology 2008;71:99-103.

http://dx.doi.org/10.1016/j.urology.2007.08.045
11. Giaquinto S, Buzzelli S, Francesco LD. Evaluation of sexual changes after stroke. J Clin Psychiatry 2003;64:302-7.

http://dx.doi.org/10.4088/JCP.v64n0312

12. Thompson HS, Assumpta R. The impact of stroke consequences on spousal relationships from the perspective of the person with stroke. J Clin Nurs 2009; 18:1803-11.

http://dx.doi.org/10.1111/j.1365-2702.2008.02694.x

13.Binder LM. Emotional problems after stroke. Stroke 1984;15:174-7.

http://dx.doi.org/10.1161/01.STR.15.1.174

14.Mota JF, Nicolato R. Qualidade de vida em sobreviventes de acidente vascular cerebral: Instrumentos de avaliaçấo e seus resultados. J Bras Psiquiatr 2008; $57: 148-56$.

15.Zétola VHF, Nóvak EM, Camargo CHF, Júnior HC, Coral P, Muzzio JA, et al. Acidente vascular cerebral em pacientes jovens: Análise de 164 casos. Arq Neuropsiquiatr 2001;59(3-B):740-5.

16.Scalzo PL, Souza ES, Moreira AGO, Vieira DAF. Qualidade de vida em pacientes com acidente vascular cerebral: Clínica de fisioterapia PUC Minas Betim. Rev Neurocienc 2010;18:139-44.

17.Lara LAS, Silva ACJSR, Romão APMS, Junqueira ARR. Abordagem das disfunçôes sexuais femininas. Rev Bras Ginecol Obstet 2008 ;30:312-21.

http://dx.doi.org/10.1590/S0100-72032008000600008

18.Lowy M, Collins S, Bloch M, Gillman M, Lording D, Sutherland P, et al. Quality of erection questionnaire correlates: Change in erection quality with erectile function, hardness, and psychosocial measures in men treated with sildenafil for erectile dysfunction. J Sex Med 2007;4:83-92.

http://dx.doi.org/10.1111/j.1743-6109.2006.00398.x

19.Marques FZC, Chedid SB, Eizerik GC. Resposta sexual humana. Rev Ciênc Méd 2008;17:175-83.

20.Abdo CH, Oliveira WM Jr, Moreira ED Jr, Fittipaldi JA. Prevalence of sexual dysfunctions and correlate conditions in a sample of brazilian women - results of the brazilian study on sexual behavior (BSSB). Int J Impot Res 2004;16:160-6.

http://dx.doi.org/10.1038/sj.ijir.3901198

21.Duggal HS, Jain R, Sinha VK, Nizamie SH. Post-encephalitic Kluver-Bucy syndrome. Indian J Pediatr 2000;67:74-6.

http://dx.doi.org/10.1007/BF02802651

22.Demerdash A, Shaalan M, Midani A, Kamel F, Bahri M. Sexual behavior of a sample of females with epilepsy. Epilepsia 1991;32:82-5.

http://dx.doi.org/10.1111/j.1528-1157.1991.tb05616.x

23.Raine A, Lencz T, Bihrle S, Casse LL, Colletti P. Reduced prefrontal gray matter volume and reduced autonomic activity in antisocial personality disorder. Arch Gen Psychiatry 2000;57:119-27.

http://dx.doi.org/10.1001/archpsyc.57.2.119

24.Antonili RS, Simões D. Abordagem fisioterapêutica nas disfunçōes sexuais femininas. Rev Neurocienc 2009;18:267-74. 